\title{
Criticality features in ULF magnetic fields prior to the 2011 Tohoku earthquake
}

\author{
By Masashi HAYAKAWA, ${ }^{* 1, * 2, * 3, \dagger}$ Alexander SCHEKOTOV,${ }^{* 2, * 4}$ \\ Stelios PotiRAKIS ${ }^{* 5}$ and Kostas EFtaXiAs ${ }^{* 6}$ \\ (Communicated by Seiya UYEDA, M.J.A.)
}

\begin{abstract}
The criticality of ULF (Ultra-low-frequency) magnetic variations is investigated for the 2011 March 11 Tohoku earthquake (EQ) by natural time analysis. For this attempt, some ULF parameters were considered: (1) $F_{h}$ (horizontal magnetic field), (2) $F_{z}$ (vertical magnetic field), and (3) $D_{h}$ (inverse of horizontal magnetic field). The first two parameters refer to the ULF radiation, while the last parameter refers to another ULF effect of ionospheric signature. Nighttime (L.T. $=3 \mathrm{am} \pm 2$ hours) data at Kakioka (KAK) were used, and the power of each quantity at a particular frequency band of $0.03-0.05 \mathrm{~Hz}$ was averaged for nighttime hours. The analysis results indicate that $F_{h}$ fulfilled all criticality conditions on March 3-5, 2011, and that the additional parameter, $D_{h}$ reached also a criticality on March 6 or 7 . In conclusion, criticality has reached in the pre-EQ fracture region a few days to one week before the main shock of the Tohoku EQ.
\end{abstract}

Keywords: earthquake (EQ), criticality, seismo-electromagnetics, natural time analysis, ULF magnetic fields, the 2011 Tohoku EQ

\section{Introduction}

Based on extensive studies during the last few decades it is generally accepted nowadays that electromagnetic phenomena appear prior to an earthquake (EQ). ${ }^{1)-4)}$ Using the amplitude data of VLF/LF (very low frequency/low frequency) transmitter signals propagating in the Earth-ionosphere waveguide during the 7-year observation, Hayakawa

*1 Hayakawa Institute of Seismo Electromagnetics Co. Ltd., UEC (University of Electro-Communications) Incubation Center, 1-5-1 Chofugaoka, Chofu, Tokyo, Japan.

*2 UEC, Advanced Wireless Communications research Center, 1-5-1 Chofugaoka, Chofu, Tokyo, Japan.

*3 Earthquake Analysis Laboratory, Information Systems Inc., \#305, Kita-aoyama 2-12-42, Minato-ku, Tokyo, Japan.

*4 Institute of Physics of the Earth, Russian Academy of Sciences, 10 Bolshaya Gruzinskaya, 123995, Moscow, Russia.

*5 Department of Electronics Engineering, Technological Education Institute (TEI) of Piraeus, 250 Thivon and P. Ralli, Aigalao, Athens GR-12244, Greece.

*6 Department of Physics, University of Athens, Panepistimiopolis, Zografos, Athens GR-15784, Greece.

Correspondence should be addressed: M. Hayakawa, Hayakawa Institute of Seismo Electromagnetics Co. Ltd., University of Electro-Communications, Incubation Center, 1-5-1 Chofugaoka, Chofu, Tokyo 182-8585, Japan (e-mail: hayakawa@ hi-seismo-em.jp). et al. $(2010)^{5)}$ established a significant statistical correlation between the perturbations in the lower ionosphere and EQs. Perturbations in the upper ionospheric $\mathrm{F}$ region observed as changes in $\mathrm{f}_{0} \mathrm{~F}_{2}$ (critical frequency of $\mathrm{F}_{2}$ layer) were also confirmed to be correlated with EQs based on long-term data. ${ }^{6)}$

Compared with these ionospheric phenomena, the number of reports is not so abundant for ULF (ultra low frequency) electromagnetic emissions, even though they were found promising for short-term EQ prediction. ${ }^{7)-10)}$ Seismogenic ULF electromagnetic waves are usually "emission or radiation" from the lithosphere as observed as intensification of ULF magnetic power. ${ }^{7-10)}$ Whereas a new phenomenon in ULF magnetic field changes is vice versa, the depression (or a decrease) in the amplitude of ULF downgoing waves of magnetospheric origin. ${ }^{11)}$

In addition to the above, there is another direction of signal processing based on the concept of critical evolution or self-organized criticality. There are a few possible methods along this line: One is fractal analysis, ${ }^{12)}$ and the other is "natural time" analysis introduced by Varotsos, ${ }^{4}$ which has been applied to time series data of a point process like SES events. ${ }^{13), 14)}$ This paper is an attempt to apply the natural time method to our ULF geomagnetic 


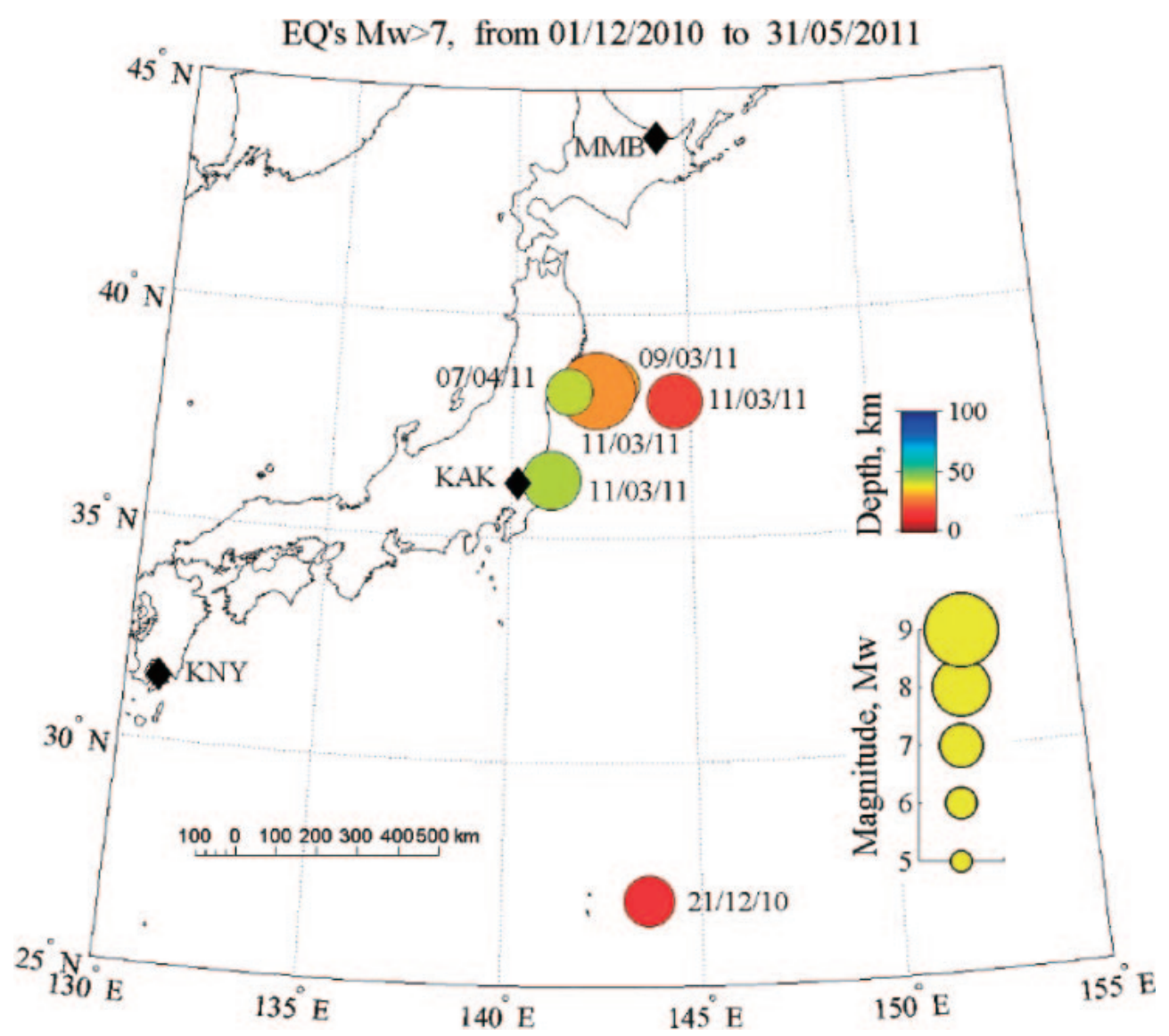

Fig. 1. Relative location of three ULF observations (KAK, MMB and KNY by black triangles) and most powerful $\left(\mathrm{M}_{\mathrm{W}}>7\right)$ and shallow (depth smaller than $100 \mathrm{~km}$ ) EQs shown by circles which occurred from December 1, 2010 to May 31, 2011. Our target EQ took place on March 11, 2011. The center of the circle is the EQ epicenter and its color indicates the depth.

data observed in Japan, especially during a period of a few months before the 2011 Tohoku EQ.

\section{ULF data and EQ information}

The ULF data were obtained from the World Data Center (WDC) for Geomagnetism, Kyoto. The data from this WDC are provided in the format of IAGA (International Association of Geomagnetism and Aeronomy) 2000, in which the magnetic field is represented by four components: Horizontal $(\mathrm{H})$, declination $(\mathrm{D})$, vertical $(\mathrm{Z})$, and total field $(\mathrm{F})$.

We use ULF data from three magnetic observatories belonging to JMA (Japan Meteorological Agency): Memambetsu (MMB) (geographic coordinates: at $\left.43^{\circ} 54^{\prime} 36^{\prime \prime} \mathrm{N}, 144^{\circ} 11^{\prime} 19^{\prime \prime} \mathrm{E}\right)$, Kakioka (KAK) (at $36^{\circ} 13^{\prime} 56^{\prime \prime} \mathrm{N}, 140^{\circ} 11^{\prime} 11^{\prime \prime} \mathrm{E}$ ), and Kanoya (KNY) (at $31^{\circ} 25^{\prime} 27^{\prime \prime} \mathrm{N}, 130^{\circ} 52^{\prime} 48^{\prime \prime} \mathrm{E}$ ) as shown by black diamonds in Fig. 1. The sampling frequency of magnetometers at these stations is $1 \mathrm{~Hz}$. Positions and magnitudes of EQs with $\mathrm{M}_{\mathrm{w}}>7$ and with depth smaller than $100 \mathrm{~km}$ are also shown by circles in the same figure. We have used seismic data from the ANSS (Advanced National Seismic System) Worldwide Earthquake Catalog. The main shock of the Tohoku EQ took place at 14:46:18 JST (UTC+9h) on March 11, 2011 with its epicenter at the geographic coordinates $\left(38^{\circ} 06^{\prime} \mathrm{N}, 142^{\circ} 52^{\prime} \mathrm{E}\right)$ as shown in Fig. 1. The magnitude was $\mathrm{M}_{\mathrm{w}}=9$ and the depth was about $20 \mathrm{~km}$. This EQ is a very typical oceanic EQ of the plate subduction type. The distance of each magnetic observatory from the EQ epicenter is $640 \mathrm{~km}$ for MMB, $300 \mathrm{~km}$ for KAK, and 1,300 km for KNY, respectively.

\section{Parameters of ULF magnetic fields to be analyzed}

The concept of "natural time" is suitable for time series data of point processes, including seismicity and DC geoelectric potential. On the other hand, ULF variations are not a point process, but they are 
continuous in nature, so that we have to think of what kind of ULF parameters should be used for the natural time analysis.

In the following, we present the results only from KAK which is closest to the EQ epicenter, because our preliminary studies indicated no significant unusual phenomena at other stations. We take, as the first step, the following procedure on ULF magnetic data to be used for the natural time analysis. To avoid industrial interference, we use ULF data only during nighttime (LT $=3 \mathrm{~h}$ a.m. \pm 2 hours). After performing spectral analysis on the raw ULF data and choosing a particular frequency range of 0.03$0.05 \mathrm{~Hz}$ (for which we have observed clear effects in $\mathrm{ULF}^{15)}$ ), we average the squared amplitude during this nighttime interval to obtain one datum per day. ${ }^{15)}$

The following ULF parameters are generated to characterize the ULF magnetic data.

(1) Horizontal magnetic field component, $\mathrm{H}$ component (power): $F_{h}=\left\langle H^{2}\right\rangle$

(2) Vertical magnetic field component, Z component (power): $F_{z}=\left\langle Z^{2}\right\rangle$

(3) Depression of horizontal magnetic field component: $D_{h}=1 /\left\langle H^{2}\right\rangle$

The parameters $F_{h}$ and $F_{z}$ are considered indicators of ULF emission from the lithosphere. The third parameter $D_{h}$ is the inverse of the horizontal magnetic field component so as to pay extensive attention to the changes in minimum values for investigating the depression of ULF waves (of magnetospheric origin) observed on the ground as the ionospheric signature. ${ }^{16)}$

Figure 2 illustrates temporal evolutions of some physical parameters: (from top to bottom) geomagnetic $\left(D_{s t}\right.$ index) and seismic $\left(K_{l s}\right.$ index $)$ activities, $F_{h}, F_{z}, P_{z / h}, D_{h}$, and ULF depression $(\delta D e p)$ as discussed in Ref. 15 , all in the same frequency range of $0.03-0.05 \mathrm{~Hz}$. The index $K_{l s}$ is a function of EQ magnitude and epicentral distance. ${ }^{3)}$ As was already shown, $\left.{ }^{15)}, 17\right)$ a significant peak in depression $(\delta D e p)$ on March 6 was observed as a precursor to the 2011 Tohoku EQ. Our glance at the temporal evolution of $F_{h}$, suggests an enhancement in the beginning (1st) of March, but we are not sure whether this is a real precursor to the EQ or not. This enhancement in $F_{h}$ looks to be correlated with a decrease in $D_{s t}$ index. It will be shown that this kind of uncertainty can be investigated with the use of natural time analysis.

\section{Natural time analysis method}

The transformation of a time-series of "events" from the conventional time domain to natural time
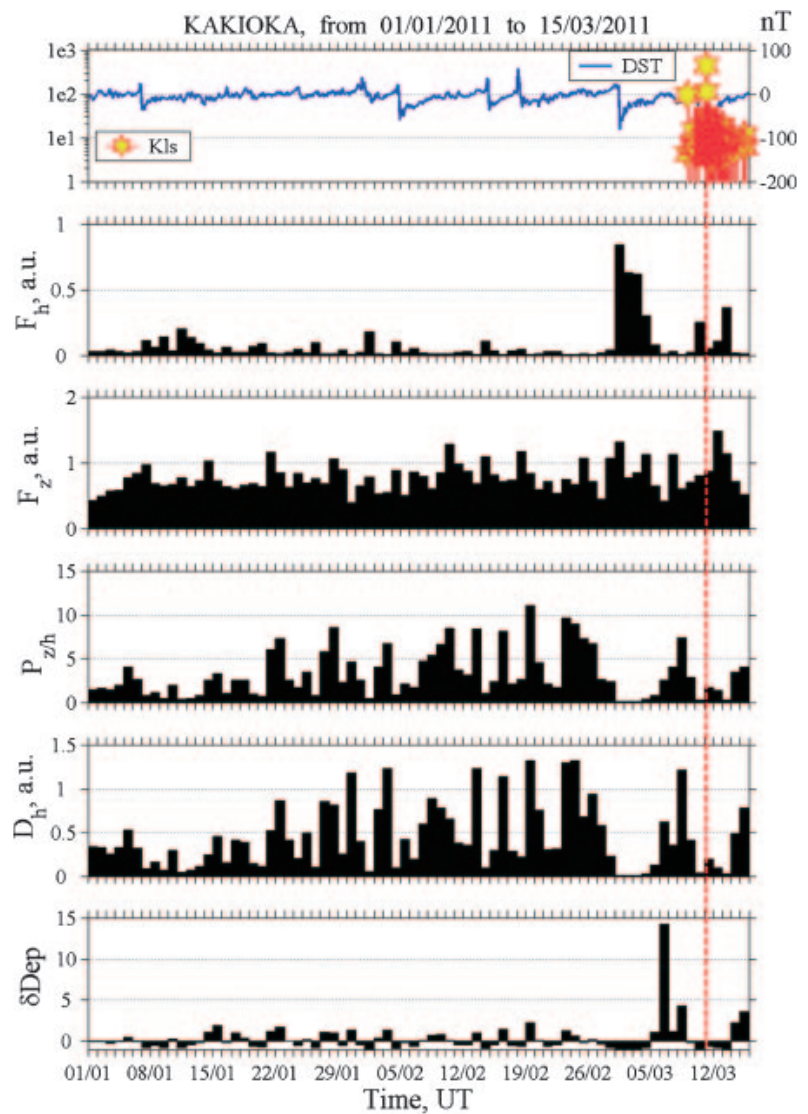

Fig. 2. Top panel: Temporal evolution of geomagnetic $\left(D_{s t}\right)$ (unit is given on the right ordinate) and seismic $\left(K_{l s}\right)$ (unit is given on the left ordinate) activities. 2nd and 3rd panels: root-meansquares of the horizontal $\left(F_{h}\right)$ and vertical $\left(F_{z}\right)$ magnetic fields in the frequency band $0.03-0.05 \mathrm{~Hz}$ (in arbitrary unit (au)), and the 4 th panel, $P_{z / h}$ (ratio of vertical and horizontal components). The 5 th and bottom panels refer to the temporal evolutions of the inverse of the horizontal component $\left(D_{h}\right)$ (in arbitrary unit (au)), and the normalized depression $\delta D e p$ as discussed in Ref. 15.

domain is performed by ignoring the time interval between events and retaining only its normalized order of occurrence. So the $k$ th event corresponds to a natural time $\chi_{k}=k / N$, where $N$ is the total number of successive events. The "energy" $Q_{k}$ of each event is also retained. Then the time series $\left(\chi_{k}, Q_{k}\right)$ is studied. A system is considered to approach criticality when the parameter $\kappa_{1}=\sum_{k=1}^{N} p_{k} \chi_{k}^{2}-\left(\sum_{k=1}^{N} p_{k} \chi_{k}\right)^{2}\left(p_{k}=\right.$ $Q_{k} / \sum_{n=1}^{N} Q_{n}$ is the energy of $k$ th event normalized by the total energy) converges to $\kappa_{1}=0.070$ and at the same time both the entropy in natural time, $S_{n t}=\sum_{k=1}^{N} p_{k} \chi_{k} \ln \chi_{k}-\left(\sum_{k=1}^{N} p_{k} \chi_{k}\right) \ln \left(\sum_{k=1}^{N} p_{k} \chi_{k}\right)$ and the entropy under time reversal, $S_{n t-}$, satisfy the condition $S_{n t}, S_{n t-}<S_{u}=(\ln 2 / 2)-1 / 4$, where $S_{u}$ stands for the entropy of a "uniform" distribution in natural time. ${ }^{18)}$ 
The criticality is considered truly achieved when the following three conditions are also satisfied: ${ }^{18)}$ (i) The "average" distance $\langle D\rangle$ between the curves of normalized power spectra $\Pi(\phi)$ of the evolving seismicity and the theoretical estimation of $\Pi(\phi)$ for $\kappa_{1}=0.070$ is smaller than $10^{-2}$, (ii) the parameter $\kappa_{1}$ approaches $\kappa_{1}=0.070$ "by descending from above", and (iii) since the underlying process is expected to be self-similar, the time of criticality does not change by varying the magnitude threshold. It may be worth mentioning that these criticality conditions, including $\kappa_{1}=0.070$, were not derived theoretically but empirically from computing $\kappa_{1}$ values for well-known phenomena and actual data of seismicity in Greece. ${ }^{18)}$

\section{Results of natural time analysis of ULF parameters}

The natural time analysis starts from 5 January 2011, following the findings of Skordas and Sarlis (2014). ${ }^{19)}$ We consider each daily value which is above a certain threshold as an event. So in our ULF cases $\left(F_{h}, F_{z}\right.$, and $\left.D_{h}\right)$, the "energy" of $k$ th event $Q_{k}$ is considered to be equal to each one of above quantities. Starting from 5 January 2011, all the parameters $\left(\kappa_{1}, S_{n t}, S_{n t-},\langle D\rangle\right.$ in Section 4) are calculated for the time-series rescaled in the natural time domain each time a new event is added. Although the selection of thresholds is arbitrary, if criticality conditions are met in close dates for more than one of the considered threshold values, then this is considered to be an indication of the validity of performed analysis.

Results of natural time analysis for different ULF parameters are presented below.

(1) $F_{h}$ (horizontal magnetic field)

Figure 3 illustrates an example of the natural time analysis results for $F_{h}$ for a particular threshold. The four critical parameters $\kappa_{1}, S_{n t}$, $S_{n t-}$ and $\langle D\rangle$ are calculated each time a new event is included in the analysis, so their evolution is seen as a function of date of new event occurrence. We found that the process was self-similar because the time of criticality did not change by changing the threshold. Then $F_{h}$ fulfilled all criticality conditions around the date of March 3, 4, or 5, 2011.

(2) $F_{z}$ (vertical magnetic field)

The corresponding figures for $F_{z}$ are not shown, because $F_{z}$ did not exhibit any criticality condition.

(3) $D_{h}$ (depression in horizontal magnetic field)

Figure 4 is again just one example of the results

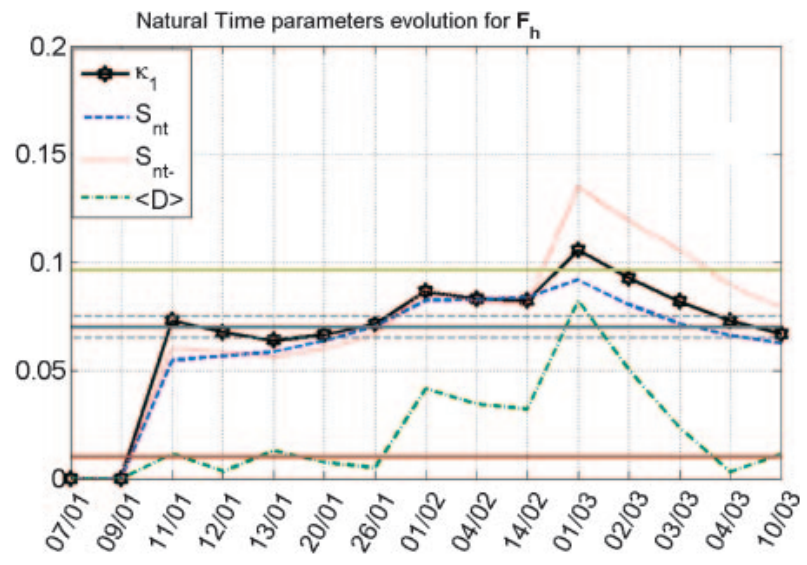

Fig. 3. Natural time analysis of the ULF electromagnetic emission quantity $F_{h}$ for the time period of 05 January through 11 March 2011: Variations of the natural time analysis parameters $\left(\kappa_{1}, S_{n t}, S_{n t-}\right.$, and $\left.\langle D\rangle\right)$ for a particular threshold.

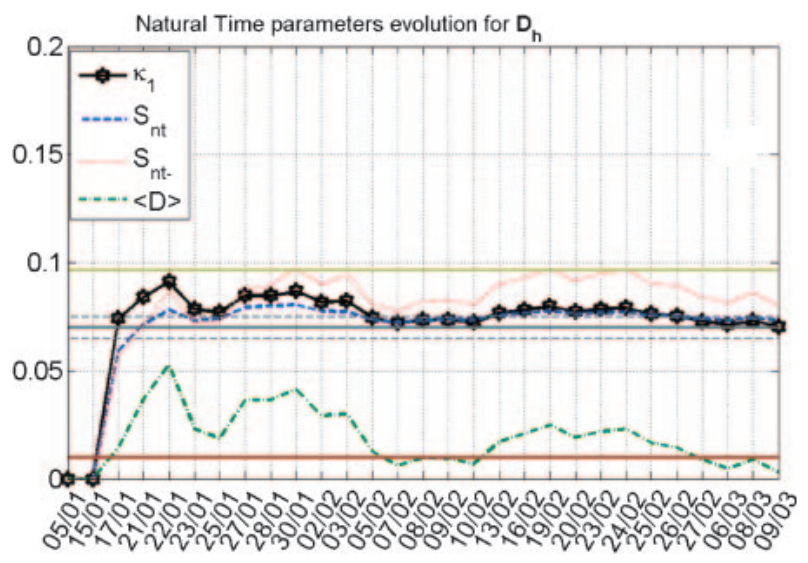

Fig. 4. Natural time analysis of the ULF electromagnetic emission quantity $D_{h}$ for the time period from 05 January to 11 March 2011: Variations of the natural time analysis parameters for a particular threshold.

of natural time analysis for the parameter $D_{h}$ for a particular threshold. We confirmed that the date of criticality did not change by changing the threshold, and so it seems that $D_{h}$ reaches a critical condition around March 6 or 7, 2011.

A question naturally arising is whether the analyzed parameters were observables of a process taking place in the Earth's crust system or any other process that could influence magnetic field variations such as magnetic storms. In Fig. 2 we find two relatively small $(-50 \mathrm{nT})$ storms on March 1 and March 11 (unfortunately this latter is masked by the plot of EQs in Fig. 2). These two magnetic storms occurred not on the same dates as the dates of 
criticality indicated by the natural time analysis of ULF parameters (March 3, 4, or 5 for $F_{h}$, March 6, 7 for $D_{h}$ ).

\section{Conclusion and discussion}

This paper is the first attempt to analyze criticality in ULF magnetic fields by means of natural time method. The results of the natural time analysis can be summarized as follows.

(1) Criticality reached a few days to one week prior to the main shock of the 2011 Tohoku EQ by fulfilling the criticality conditions for the two parameters $F_{h}$ and $D_{h}$, but not for $F_{z}$.

(2) These critical dates seem to have no association with geomagnetic activity.

First, we discuss the lithospheric aspect of the ULF parameters, $F_{h}$, and $F_{z}$. It is not clear why we have detected criticality only in $F_{h}$, but not in $F_{z}$. Tentatively, we consider as follows. The 2011 Tohoku EQ was the most powerful EQ ever recorded to have hit Japan, and Hirose et al. $(2011)^{20)}$ reported that the hypocentral region of this EQ is extended from offshore Iwate prefecture to offshore Ibaraki prefecture, with a length of $500 \mathrm{~km}$ and a width of $200 \mathrm{~km}$. From the huge area of the rupture region, it may not be unreasonable to consider that the corresponding pre-fracture region is also very wide, even extending to our ULF observation point of KAK. Whatever the mechanism of electrification in the source region, dynamic processes in association with pre-EQ microfracture can lead to the generation of current systems. ${ }^{21), 22)}$ Our finding of the criticality at KAK only in $F_{h}$, but not in $F_{z}$, may be explained by the relative position of the current source and observatory. When using the method as used in Kawate et al. (1998), ${ }^{23)}$ we can infer that the source current, at least, responsible for the seismogenic ULF radiation observed at KAK, might have been located in an area relatively close to KAK, for example, offshore Ibaraki prefecture.

Next we move on to the ionospheric ULF phenomena. Criticality was observed on March 6 and 7 in the temporal evolution of critical parameters for $D_{h}$, which is in agreement with the conclusion of Schekotov et al. (2013). ${ }^{15)}$ They found a depression in ULF magnetic field intensity on the ground (as shown in the bottom panel of Fig. 2), which may be satisfactorily interpreted in terms of an enhanced absorption of downgoing Alfvén waves through the perturbed lower ionosphere. ${ }^{17)}$ It seems that the preEQ criticality has taken place in a wide area of the lithosphere, and the criticality is also recognized in the lower ionosphere with a small time difference of one day or so. The area of lower ionospheric perturbation as sensed by the ULF magnetic field depression is found to be much wider ${ }^{15), 17)}$ than such a localized area (radius less than $100 \mathrm{~km}$ ) for the lithospheric ULF radiation observed at KAK. So the information on ionospheric perturbations derived from $D_{h}$ is likely to reflect the criticality taking place on March 6 and 7 in the lithosphere over a wider area of the rupture region in the Pacific ocean. The difference in criticality dates for $F_{h}$ and $D_{h}$ is probably due to slightly different dates for criticality at a relatively localized region near KAK and at a much wider pre-EQ fracture region.

\section{Acknowledgements}

We would like to express our sincere thanks to the unknown two referees and Prof. S. Uyeda for their valuable comments.

\section{References}

1) Hayakawa, M. and Molchanov, O.A. (eds.) (2002) Seismo Electromagnetics: Lithosphere-Atmosphere-Ionosphere Coupling. TERRAPUB, Tokyo.

2) Pulinets, S. and Boyarchuk, K. (2004) Ionospheric Precursors of Earthquakes. Springer, Berlin.

3) Molchanov, O.A. and Hayakawa, M. (2008) SeismoElectromagnetics and Related Phenomena: History and Latest Results. TERRAPUB, Tokyo.

4) Varotsos, P.A. (2005) The Physics of Seismic Electric Signals. TERRAPUB, Tokyo.

5) Hayakawa, M., Kasahara, Y., Nakamura, T., Muto, F., Horie, T., Maekawa, S., Hobara, Y., Rozhnoi, A., Solovieva, M. and Molchanov, O.A. (2010) A statistical study on the correlation between lower ionospheric perturbations as seen by subionospheric VLF/LF propagation and earthquakes. J. Geophys. Res. 115, A09305, doi:10.1029/ 2009JA015143.

6) Liu, J.Y. (2009) Earthquake precursors observed in the ionospheric F-region. In Electromagnetic Phenomena Associated with Earthquakes (ed. Hayakawa, M.). Transworld Research Network, Trivandrum, India, pp. 187-204.

7) Kopytenko, Yu.A., Matiashvily, T.G., Voronov, P.M., Kopytenko, E.A. and Molchanov, O.A. (1990) Discovering of ultra-low-frequency emissions connected with Spitak earthquake and its aftershock activity on data of geomagnetic pulsations observations at Dusheti and Vardzija. IZMIRAN, Preprint No. 3a(888).

8) Molchanov, O.A., Kopytenko, Yu.A., Voronov, P.M., Kopytenko, E.A., Matiashvili, T.G., Fraser-Smith, A.C. and Bernardi, A. (1992) Results of ULF magnetic field measurements near the epicenters of the Spitak $(\mathrm{M}=6.9)$ and the Loma-Prieta $(\mathrm{M}=7.1)$ earthquakes: Comparative 
analysis. Geophys. Res. Lett. 19, 1495-1498.

9) Fraser-Smith, A.C., Bernardi, A., McGill, P.R., Ladd, M.E., Helliwell, R.A. and Villard, O.G. Jr. (1990) Low-frequency magnetic field measurements near the epicenter of the Ms 7.1 Loma Prieta earthquake. Geophys. Res. Lett. 17, 14651468.

10) Hayakawa, M., Kawate, R., Molchanov, O.A. and Yumoto, K. (1996) Results of ultra-low-frequency magnetic field measurements during the Guam earthquake of 8 August 1993. Geophys. Res. Lett. 23, 241-244.

11) Schekotov, A., Fedorov, E., Molchanov, O.A. and Hayakawa, M. (2013) Low frequency electromagnetic precursors as a prospect for earthquake prediction. In Earthquake Prediction Studies; Seismo Elecromagnetics (ed. Hayakawa, M.). TERRAPUB, Tokyo, pp. 81-99.

12) Hayakawa, M., Itoh, T. and Smirnova, N. (1999) Fractal analysis of ULF geomagnetic data associated with the Guam earthquake on August 8, 1993. Geophys. Res. Lett. 26 (18), 2797-2800.

13) Varotsos, P., Sarlis, N.V., Skordas, E.S., Uyeda, S. and Kamogawa, M. (2011) Natural time analysis of critical phenomena. Proc. Natl. Acad. Sci. U.S.A. 108, 11361-11364, doi:10.1073/pnas.1108138108.

14) Uyeda, S., Kamogawa, M. and Tanaka, H. (2009) Analysis of electrical activity and seismicity in the natural time domain for the volcanic-seismic swarm activity in 2000 in the Izu Island region, Japan. J. Geophys. Res. 114, B02310, doi:10.1029/ 2007JB005332.

15) Schekotov, A., Fedorov, E., Hobara, Y. and Hayakawa, M. (2013) ULF magnetic field depression as a possible precursor to the $2011 / 3.11$ Japan earthquake. J. Atmos. Electr. 33 (1), 41-51.

16) Schekotov, A., Molchanov, O.A., Hattori, K., Fedorov, E., Gladyshev, V.A., Belyaev, G.G., Chebrov, V., Sinitsin, V., Gordeev, E. and Hayakawa, M. (2006) Seismo-ionospheric depression of the ULF geomagnetic fluctuations at Kamchatka and Japan. Phys. Chem. Earth 31, 313-318.
17) Hayakawa, M., Rozhnoi, A., Solovieva, M., Hobara, Y., Ohta, K., Schekotov, A. and Fedorov, E. (2013) The lower ionospheric perturbation as a precursor to the 11 March 2011 Japan earthquake. Geomatics, Natural Hazards and Risk 4 (3), 275287, doi:10.1080/19475705.2012.751938.

18) Varotsos, P.A., Sarlis, N.V., Skordas, E.S., Tanaka, H.K. and Lazaridou, M.S. (2006) Entropy of seismic electric signals: Analysis in the natural time under time reversal. Phys. Rev. E 73, 031114, doi:10.1103/PhysRevE.73.031114.

19) Skordas, E.S. and Sarlis, N.V. (2014) On the anomalous changes of seismicity and geomagnetic field prior to the $2011 \mathrm{M}_{\mathrm{w}} 9.0$ Tohoku earthquake. J. Asian Earth Sci. 80, 161-164, doi:10.1016/ j.jseaes.2013.11.008.

20) Hirose, F., Miyaoka, K., Hayashimoto, N., Yamazaki, T. and Nakamura, M. (2011) Outline of the 2011 off the Pacific coast of Tohoku earthquake $\left(\mathrm{M}_{\mathrm{w}} 9.0\right)$-seismicity: foreshocks, mainshock, aftershocks, and induced activity. Earth Planets Space 63, 513-518, doi:10.5047/ eps.2011.05.019.

21) Molchanov, O.A. and Hayakawa, M. (1995) Generation of ULF electromagnetic emissions by microfracturing. Geophys. Res. Lett. 22, 30913094.

22) Vallianatos, F. and Tzanis, A. (1999) A model for the generation of precursory electric and magnetic fields associated with the deformation rate of the earthquake focus. In Atmospheric and Ionospheric Electromagnetic Phenomena Associated with Earthquakes (ed. Hayakawa, M.). TERRAPUB, Tokyo, pp. 287-306.

23) Kawate, R., Molchanov, O.A. and Hayakawa, M. (1998) Ultra-low-frequency magnetic fields during the Guam earthquake of 8 August 1993 and their interpretation. Phys. Earth Planet. Inter. 105, $239-248$.

(Received Sep. 19, 2014; accepted Nov. 27, 2014) 